\title{
Perception of vehicle speed as a function of vehicle size
}

\author{
ROBERT J. HERSTEIN \\ Maryland State Highway Administration, Hanover, Maryland \\ and \\ MARGARET L. WALKER \\ Towson State University, Baltimore, Maryland
}

(Michael H. Figler, Sponsor)

\begin{abstract}
Previous research suggests that an observer's ability to detect the motion of an object increases as the size of the object increases. Anecdotal evidence suggests that the opposite is true. In this experiment, 84 subjects watched a videotape of either a motorcycle, a Jeep Cherokee, or a singleaxle dump truck approaching at either $40 \mathrm{~km} / \mathrm{h}$ or $80 \mathrm{~km} / \mathrm{h}$ for $4 \mathrm{sec}$. The subjects were then asked to estimate the time it would take for the vehicle on the tape to hit them if it continued at the same speed. The subjects could accurately discern the difference in speeds, but no effect of vehicle size was found on the subjects' estimates of the time to collision. Previous researchers used approaching objects with a wide variance in size placed close to the observers. In this experiment, the vehicles were relatively similar in size and were observed from a long distance.
\end{abstract}

Over the last 6 years, there have been about $6,000 \mathrm{col}-$ lisions per year at highway-rail grade crossings throughout the United States. These accidents have resulted in about 3,000 casualties, including about 600 fatalities, per year (Federal Railroad Administration [FRA], 1989). In most highway-rail accidents, the driver of the highway vehicle is considered to be at fault. The train cannot deviate from its path on the tracks to avoid a collision. In addition, the train cannot stop very quickly, needing as long as $3 \mathrm{~km}$ to stop under the most adverse conditions. In about $72 \%$ of these collisions, a train struck the highway user (FRA, 1989). These collisions are attributed to deliberately risky behavior, impairment of senses and judgment by drugs or other causes, or the catchall driver error.

An important variable in driver error that contributes to accidents with trains is the driver's inability to judge accurately the time it will take the train to reach the crossing. Liebowitz (1985) isolated three visual/perceptual variables that prevent highway users from safely determining the time to a collision. The first variable is the inverse relationship between size and apparent speed. Specifically, the larger an object is, the slower it appears to be moving.

The second variable is the phenomenon in which twodimensional cues give the illusion of depth. An example of this is the way in which the two parallel rails of a railroad track appear to meet in the distance. Drivers use this apparent meeting point as a scale to measure distance.

Correspondence should be addressed to R. J. Herstein, Statewide Studies Section, Maryland State Highway Administration, 7491 Connelley Dr., Hanover, MD 21076.
However, this phenomenon causes the illusion that an approaching vehicle is much farther away than it actually is.

The third variable is the principal of looming. One cue to the speed of an approaching object is its increase in apparent size. The relationship between this increase in apparent size and the object's distance from the observer is hyperbolic (see Figure 1). Looming is the sudden increase in apparent size as the object gets close to the observer. The nonlinearity of this function can be misleading in determining the time to a collision.

The use of the apparent change in size of an approaching object as a cue to determine its direction of movement and velocity has been well established. Ittelson (1951) determined the relationship between real and apparent movement of an object when the size of the object changed with the use of a two-alley arrangement. No correlation between the size of the object and the difference between reported and actual movement was found.

A series of experiments was conducted by Baker and Steedman (1961) to test subjects' abilities to detect forward or backward motion of a target object. The direction of movement was detected when the visual angle increased or decreased by about $2 \%$ regardless of speed (Baker \& Steedman, 1961). In a related study, Steedman and Baker (1962) held luminance constant and varied angular size from $0.025^{\circ}$ to $1^{\circ}$. The change in angular size needed to detect the direction of movement increased as the angular size decreased.

Schiff (1965) performed several experiments to determine if looming elicited an avoidance reaction in humans. The subjects correctly reported all stimulus events as the movement of an object of constant size and correctly described each of the objects, noting the direction of move- 


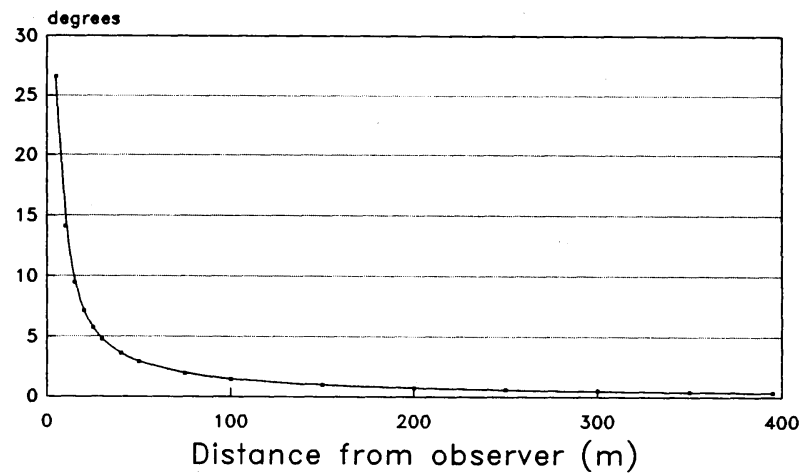

$\rightarrow$ Visual Angle

Figure 1. Visual angles of an object $2.5 \mathrm{~m}$ high.

ment, in each trial. The subjects also correctly reported the relative velocity in each group of trials.

The purpose of the present experiment was to determine the nature of the relationship between the size of an approaching vehicle and a driver's perception of its velocity. In addition, the effects of approach speed and the interaction between approach speed and size on perceived speed were examined.

\section{METHOD}

\section{Subjects}

The subjects were 84 students at Towson State University. There were 31 males and 53 females ranging in age from 18 to 47 . All of the subjects were licensed drivers. Licensing agencies require minimum corrected visual acuity of 20/40. The subjects who were required by license restriction to wear corrective lens were asked to wear these devices while participating in the test. Each subject participated in only one treatment condition in a $2 \times 3$ fully randomized functional design.

\section{Apparatus}

The subjects were seated and watched a videotape on a monitor from the vantage point of a driver looking through the windshield of an automobile. The sound was turned off because auditory cues were not germane to this experiment. The film showed one of three vehicles approaching at one of two speeds.

The approaching vehicles were a 1984 Yamaha RZ350 motorcycle, a 1990 Jeep Cherokee, and a 1981 Ford K747 single-axle dump truck. The cross-sectional areas of these vehicles were about $1.2 \mathrm{~m}^{2}, 2.4 \mathrm{~m}^{2}$, and $6.2 \mathrm{~m}^{2}$, respectively.

All of these vehicles were yellow with black trim, and the motorcycle rider wore a black jacket. Also, the headlights were turned off on all three vehicles. All of the headlights were of different sizes and shapes and were arrayed in different patterns on each vehicle. Turning them off eliminated the possibility of the optical expansion of the headlights providing a cue to the vehicle's speed.

Each vehicle traveled at a constant speed toward the video camera. Filming began when the vehicle was $2 \mathrm{sec}$ away from a point $222 \mathrm{~m}$ from the camera and continued for $4 \mathrm{sec}$. Each vehicle was filmed traveling $40 \mathrm{~km} / \mathrm{h}$ and $80 \mathrm{~km} / \mathrm{h}$. At these speeds, it would take the vehicles $18 \mathrm{sec}$ and $8 \mathrm{sec}$, respectively, to reach the camera. Speed was verified to $\pm .6 \mathrm{~km} / \mathrm{h}$ with a Decatur Electronics Range Master X-band radar unit, Model RM715. The camera was mounted on a tripod $1.4 \mathrm{~m}$ above the ground, a height at about the eye level of a driver in a modern compact car.

The subjects were asked to record their responses to the videotape on a questionnaire. The primary response that was requested was the number of seconds that would elapse from the time the film stopped until the time when the vehicle on the tape would collide with them. In addition, the subjects were asked if they would walk or drive across the highway in the situation that they had observed. The questionnaire also contained instructions and demographic questions for the subjects.

\section{Procedure}

The subjects were randomly assigned to observe one tape showing one vehicle at one speed. After completing an informed consent form, they were given the questionnaire and asked to follow the directions on it. They were orally admonished to read the form completely before proceeding with the test. No other instructions were given.

After the subjects indicated that they had completed the upper portion of the form and had read the questions on the lower portion, they were seated in front of the video monitor at a distance of about $0.7 \mathrm{~m}$, and the tape was started. When the tape ended, they completed the form. The tape was then reset for the next subject.

\section{RESULTS}

The subjects were asked to estimate the time, in seconds, it would take for the vehicle presented on the tape to collide with them if it continued to travel at the same speed. A two-factor analysis of variance ( 3 vehicles, 2 speeds) was conducted on the subjects' predictions of the time to collision. The apparent difference between the predicted times for each speed was found to be significant $[F(1,79)=10.33, p<.005]$.

The mean predicted times to collision by vehicle type appeared to be virtually identical, and no significant effect was found for vehicle type $[F(2,79)=.139, p>.005]$. A nonsignificant interaction between vehicle type and speed was found. The motorcycle's times tended to be higher than the other vehicles' times at $40 \mathrm{~km} / \mathrm{h}$ and lower than the other vehicles' times at $80 \mathrm{~km} / \mathrm{h}[F(2,79)=2.48$, $p>.005]$.

In addition to asking the subjects to estimate the time to a collision, we asked them if they would walk across the highway in the situation that they had just seen. The subjects were more willing to walk across the highway in front of a vehicle approaching at $40 \mathrm{~km} / \mathrm{h}$ than they were in front of one approaching at $80 \mathrm{~km} / \mathrm{h}$. A chi-square test was conducted on the data for each speed separately and on both speeds combined to determine if the number of subjects willing to cross the highway in front of a particular vehicle was different from that expected by chance. The difference was not significant for $80 \mathrm{~km} / \mathrm{h}$ $\left[\chi^{2}(2)=5.939, p>.05\right]$ and for both speeds combined $\left[\chi^{2}(2)=2.275, p>.05\right]$. It was significant for $40 \mathrm{~km} / \mathrm{h}$ $\left[\chi^{2}(2)=7.93, p<.025\right]$.

The subjects were also asked if they would drive across the highway in the situation that they had just seen. The subjects were also more willing to drive across the highway in front of a vehicle approaching at $40 \mathrm{~km} / \mathrm{h}$ than they were in front of one approaching at $80 \mathrm{~km} / \mathrm{h}$; however, this difference was not significant $\left[\chi^{2}(1)=1.429, p>.05\right]$.

\section{DISCUSSION}

Previous research presents three possible relationships between subjects' perceptions of an object's speed and its size. Steedman and Baker (1962) suggested that subjects perceive larger objects to be traveling faster than smaller ones when both are traveling at the same velocity. 
Anecdotal evidence cited in Liebowitz (1985) suggested that the reverse is true. Ittelson (1951) implied that the size of an object has no effect on a subject's ability to judge its speed. In the present experiment, the size of the approaching vehicle had no demonstrable effect on the subjects' ability to judge its speed.

The relationship between an object's increase in apparent size and its distance from the observer is hyperbolic (see Figure 1). The closer an object is to an observer, the faster its apparent size changes. Steedman and Baker (1962) used a light varying in area from $0.09 \mathrm{~cm}^{2}$ to $140.1 \mathrm{~cm}^{2}$, with movement starting $7.6 \mathrm{~m}$ from the subject. The light varied in beginning angular size from $0.025^{\circ}$ to $1^{\circ}$. The largest object was 1,557 times larger than the smallest, and the largest visual angle was 40 times larger than the smallest.

Ittelson (1951) placed his approaching objects at a mean distance of $2.7 \mathrm{~m}$ from his subjects. The objects varied in size from $14 \mathrm{~cm}^{2}$ to $203 \mathrm{~cm}^{2}$. The largest object was 14 times the size of the smallest. At this distance, with this variation in size, no effect was found for object size.

In the present experiment, the approaching vehicles varied in size from $1.2 \mathrm{~m}^{2}$ to $6.2 \mathrm{~m}^{2}$. The largest vehicle was 5 times the size of the smallest vehicle. The vehicles were viewed at a mean distance of $222 \mathrm{~m}$. The visual angle changed from $0.35^{\circ}$ to $0.52^{\circ}$ for the motorcycle at $80 \mathrm{~km} / \mathrm{h}$. It changed from $0.54^{\circ}$ to $0.81^{\circ}$ for the dump truck at $80 \mathrm{~km} / \mathrm{h}$. At this distance, with objects this similar in apparent size, the difference in the change in apparent size between the three vehicles is less than that which occurred in Steedman and Baker (1962).

The perception of an inverse relationship between size and apparent speed reported by Liebowitz (1985) is most noticeable when objects are moving parallel to one another or pass the observer in rapid succession. In the present experiment, the subjects observed only one vehicle. They did not have another vehicle for comparison. Therefore, the perception of size as inversely proportional to apparent speed was not elicited.

In line with what Schiff (1965) demonstrated, the present subjects could perceive the difference in speeds. The mean estimated time to collision was significantly lower for $80 \mathrm{~km} / \mathrm{h}$ than it was for $40 \mathrm{~km} / \mathrm{h}$. However, the subjects demonstrated an inability to accurately estimate the time to collision. Of 84 trials, there were only two correct estimates. There were over twice as many underestimates as overestimates. The subjects were no more likely to overestimate or underestimate for any particular vehicle, which is consistent with the other findings of the experiment.

Baker and Steedman (1961) demonstrated that the speed of an approaching object had no effect on the observer's ability to judge its speed. If there had been an effect of speed, it would have been found in the analysis of the difference between the subjects' estimates of the time to collision and the actual time. No effect of speed was found. The subjects were no more likely to overestimate or underestimate for either speed.

Humans generally seem to have a great deal of difficulty in estimating measurements against a standard, which was why subjects were asked if they would walk or drive across the intersection they had seen. It was believed that even if subjects could not accurately estimate the actual time to collision, they could use some other process to determine whether they could safely cross an intersection when a vehicle was approaching.

The speed at which pedestrians walk is fairly uniform for people in the age group represented by the subjects. Therefore, the controlling factors in their decision to cross the highway in a particular situation were their tendency to take risks and their perception of the time it took the approaching vehicle to reach them. As expected, the subjects were more likely to cross in front of a vehicle approaching at $40 \mathrm{~km} / \mathrm{h}$ than they were with one approaching at $80 \mathrm{~km} / \mathrm{h}$.

The subjects' willingness to walk across the intersection gave a more reliable indication of their perception of the time it took an approaching vehicle to reach them than did their willingness to drive across it. There is much more variability in the time it takes drivers to traverse an intersection. The drivers' tendency to take risks, the acceleration properties of their vehicles, and their ability to make full use of those properties control the time it takes to accelerate from a stop and cross the highway. With this subject variability, it was not surprising that no effect was found for vehicle type. Although this does support the results found in the time estimations and the subjects' willingness to walk across the intersection, little credence should be placed on it due to subject variability.

In summary, this experiment demonstrated that the size of an approaching vehicle may not have had the effect on a driver's ability to estimate its speed that other researchers thought it had. The subjects could consistently determine the relative speed of different approaches but could not accurately estimate the time to collision. They were not better able to estimate the approach speed of one size of vehicle over another. These perceptual limitations may, in fact, account for the high number of highway collisions that occur annually.

\section{REFERENCES}

Baker, C. A., \& Steedman, W. C. (1961). Perceived movement in depth as a function of luminance and velocity. Human Factors, 3, 166-173.

Federal Railroad Administration. (1989). Rail-Highway Crossing Accident/Incident and Inventory Bulletin, No. 11, Calendar Year 1988. Washington, DC: U.S. Department of Transportation.

ItTELSON, W. H. (1951). Size as a cue to distance: Radial motion. American Journal of Psychology, 64, 188-202.

Liebowitz, H. W. (1985). Grade crossing accidents and human factors engineering. American Scientist, 73, 558-562.

SCHIFF, W. (1965). Perception of impending collision: A study of visually directed avoidant behavior. Psychological Monographs: General and Applied, 79(Serial No. 604).

Steedman, W. C., \& Baker, C. A. (1962). Perceived movement in depth as a function of stimulus size. Human Factors, 4, 349-354.

(Manuscript received June 17, 1993.) 